\title{
Observational study of fetomaternal outcome in pregnancy with COVID-19 infection
}

\author{
Pragyan Dash*, Sobhan K. Padhi, Rabindra N. Behera
}

Department of Obstetrics and Gynecology, Hi-Tech Medical College and Hospital, Bhubaneswar, Odisha, India

Received: 10 January 2022

Revised: 06 February 2022

Accepted: 07 February 2022

\section{*Correspondence:}

Dr. Pragyan Dash,

E-mail: pragyandash89@gmail.com

Copyright: ( ) the author(s), publisher and licensee Medip Academy. This is an open-access article distributed under the terms of the Creative Commons Attribution Non-Commercial License, which permits unrestricted non-commercial use, distribution, and reproduction in any medium, provided the original work is properly cited.

\begin{abstract}
Background: According to World Health Organization (WHO) COVID-19 is declared as an infection of international health emergency on 11 March 2020. It is not clear whether clinical characteristics of pregnant women with COVID19 infection differ from those of non-pregnant women and whether it aggravates its symptoms.

Methods: This is a prospective observational study of 75 cases based on compiled clinical data of pregnant women with COVID-19 infection admitted to Hi-Tech Medical College from June 2020 to July 2021. All laboratory confirmed positive cases were included.

Results: The most common symptoms reported was fever (24\%) followed by myalgia, cough and shortness of breath, while $60 \%$ patients were asymptomatic. there were total 57 deliveries, out of which 46 were live birth. The incidence of preterm birth was $26.3 \%$. Maternal mortality was maximum between 25-34 weeks of GA. 96.9\% of new-born were tested for SARS CoV-2 and only 10 were found to be positive $(17.5 \%)$.

Conclusions: At present there is no evidence regarding increased risk of pregnant women succumbed to COVID-19 infection and experience severe pneumonia. The risk of preterm birth and spontaneous abortion not increased as reported in this study but shows possibilities of vertical transmission. COVID-19 infection in pregnancy with or without any comorbidities can cause complication to both mother and fetus irrespective of treatment. We aim to evaluate the association between SARS CoV-2 infection during pregnancy and adverse pregnancy outcomes.
\end{abstract}

Keywords: COVID-19, SARS CoV-2, Pregnancy, Pneumonia, Preterm

\section{INTRODUCTION}

Atypical pneumonia known as coronavirus disease (COVID-19), which is caused by the severe acute respiratory syndrome coronavirus-2 (SARS-CoV-2) virus, is highly infectious and is currently spreading rapidly around the globe. Since the emergence of SARS-CoV-2 in Wuhan, Hubei Province, China, during December 2019, it has caused thousands of morbidities and mortalities around the globe. ${ }^{-}$It was declared a global pandemic in March $2020 .^{2}$ Pregnant women do not appear more likely to contract the infection than the general population, but with alterations in hormone levels and decreased functional residual capacity due to uterus and slightly immunocompromised (which include changes to $\mathrm{T}$ lymphocyte immunity) in pregnancy there might be greater risk for both the mother and fetus. ${ }^{4}$ Many studies have focused on infected patients from the general population; however, details related to pregnancy outcomes of women with COVID-19 are scarce fetuses may be exposed to SARS-CoV-2 during critical periods of fetal development. $\underline{3}$ COVID-19 pandemic is causing concern also for the management and outcome of COVID-19 positive pregnant women and their offspring, as reported cases are rare.

In general, based on the data collected fever, cough, myalgia were the most common clinical features, while abnormal laboratory parameters includes 
lymphocytopenia and raised C-reactive protein (CRP). Bilateral pneumonia and ground glass opacity were the most common radiological parameters. patients were treated with oxygen therapy, antibiotics, antivirals when necessary and other drugs as per Indian Council of Medical Research (ICMR) guidelines. Middle East respiratory syndrome coronavirus-2 (MERS CoV-2) was the leading cause of severe cases in infected pregnant women. Pregnant women infected with COVID-19 infection are at increased risk of adverse obstetrical outcome as compared to general population. There is little evidence about the possible impact of COVID in early pregnancy (up to 12 week gestation). Seasonal influenza has been associated with higher rates of miscarriage and no clear evidences available whether this is same with COVID-19 infection. $\underline{6}$ In late pregnancy (>24 weeks gestation), we can expect that COVID-19 infection could cause increased rates of adverse pregnancy outcomes such as fetal growth restriction, preterm birth and perinatal mortality.., $7-$ The infection outcomes are mainly associated with increased rate of caesarean delivery, preterm birth, intensive care admission, pre-eclampsia, miscarriage, fetal distress and perinatal death. Women with pre-existing comorbidities, older age, high body mass index (BMI) and women of black, Asian and minority ethnic groups (BAME) may be at increased risk of hospitalization or severe outcome. . $^{5,9}$ With regard to vertical transmission evidences now suggests that vertical transmission is probable, although the proportion of pregnancy affected and significance to the neonates has yet to be determined. Presently there is no documented evidences of COVID-19 infection in vaginal secretion or breast milk.

Against the backdrop of this pandemic, it becomes imperative to study the effects of this infection on pregnancy and its outcome. Hence, this study was undertaken to evaluate the effects of COVID-19 infection (SARS-CoV-2, MERS-CoV, and SARS-CoV) on the maternal morbidity and mortality, the course of labour, mode of delivery, as well as the neonatal outcome. Therefore, we conducted a prospective, observational study in Hi Tech Medical College and Hospital, Bhubaneswar (Odisha), India to know the association between COVID-19 infection and maternal and neonatal outcomes in pregnant women.

\section{Aims and objectives of the study}

Aims and objectives of the study include maternal outcome in pregnancy with COVID-19 infection, and to study fetal outcome in COVID-19 positive mothers.

\section{METHODS}

\section{Place of study}

The study was conducted at the department of obstetrics and gynaecology, Hi-Tech Medical College and Hospital, Bhubaneswar, Odisha, India.

\section{Study design}

It was a tertiary care hospital based observational study.

\section{Period of study}

The study was conducted from June 2020 - July 2021.

\section{Inclusion criteria}

All pregnant women with COVID-19 positive result in rapid antigen test or by reverse transcriptase-polymerase chain reaction (RT-PCR) test admitted to covid obstetrics and gynaecology ward and COVID labour room.

\section{Exclusion criteria}

All pregnant females admitted to $\mathrm{HMCH}$ obstetrics and gynaecology ward with negative COVID-19 report, and patients who don't consent for the study were excluded.

\section{Consent of patient}

Consent for all cases have to be taken before in their own language.

It is an observational study from June 2020 to July 2021. All the data to be tabulated in Microsoft excel sheets and calculation to be done by using appropriate statistical formulas, using percentage and mean. N-95 mask, splash resistant face shield, goggles were used by all the health care professionals in labour wards and operation theatres. Separate premises were assigned as donning and doffing areas.

Complete epidemiologic history, obstetric history, clinical symptoms, signs, specific obstetric condition, laboratory and radiologic investigations, treatment measures, complications and outcome data were collected from electronic medical records software ARCUS and curated within customized data collection format. All laboratory investigations and radiologic assessments were performed according to the clinical care needs of the present pregnancy. Laboratory investigations included complete blood cell count (CBC), liver function test (LFT), renal function test (RFT), C-reactive protein (CRP), serum lactate dehydrogenase (LDH), thyroid function test (TFT) and HbA1C. Data on pregnancy and neonatal outcome, including gestational age at delivery, mode of delivery, and indication for caesarean delivery, complications, neonatal birthweight and neonatal intensive care unit (NICU) admission were analyzed. Neonatal pharyngeal and nasopharyngeal swab samples were collected immediately after delivery in the NICU and tested for SARS-CoV-2 using RT-PCR. Sample collection, processing, and laboratory testing were complied with World Health Organization (WHO) guidance. 


\section{RESULTS}

The characteristics and outcomes of the study population of 75 laboratory confirmed COVID-19 positive cases are demonstrated.

As per our study 2 patients were less than 20 years of age $81.3 \%$ (61) patients belong to age group of $21-35$ years with a mean age of 28 years. 12 patients were of $>35$ years.

In Table 1, 12 patients belong to $>35$ year out of which 7 were symptomatic of which 5 patients succumbed which is $71.4 \%$ of that group. 61 patients belong to $21-35$ year of age grp out of which 22 were symptomatic. Only 2 patients were critical and died in this age group which is $9 \%$. Only 1 patient <20 year age group admitted with severe symptoms and died.

9 patients admitted in $1^{\text {st }}$ trimester $(<12$ weeks $), 8$ were in $2^{\text {nd }}$ trimester (12-24 weeks), 19 belong to $25-34$ weeks gestation. Maximum number of patients $52 \%$ (39) belong to $>34$ weeks of gestation. As per Table 2 out of 39 patients in $>34$ weeks gest group only 14 were asymptomatic i.e. $35.8 \%$, whereas 9 out of 19 patients in 25-34 weeks GA were symptomatic i.e. $47.3 \%$. 4 out of 9 patients $(44.4 \%)$ in $1^{\text {st }}$ trimester and 3 out of $8(37.5 \%)$ patients of $2^{\text {nd }}$ trimester were symptomatic.

As per the study, the most common symptoms at presentation were fever in $60 \%(18 / 30)$ and myalgia in $50 \%$ (15/30). Other reported symptoms were cough in
$33.34 \%(10 / 30)$, shortness of breath in $26.67 \%(8 / 30)$ while $60 \%(45 / 75)$ were asymptomatic.

Out of 75 patients antenatal patients admitted with COVID positive report 8 were managed conservatively, 9 underwent suction and evacuation, 57 patients delivered and laparotomy performed on 1 patient due to ectopic pregnancy. There were 11 cases with intrauterine device (IUD). Present study reported 46 live births. The incidence of missed abortion was $12 \%(9 / 75)$.

Out of 57 patients who delivered, lower segment caesarean section (LSCS) was performed as per under mentioned indications. 12 patients due to scar tenderness with previous LSCS, 4 due to non-progress of labour, 2 were obstructed, 6 patients for cephalon pelvic disproportion, 2 for severe oligohydramnios, 2 for severe pre-eclampsia with unfavourable cervix, 2 for placenta previa. As the chart shows, all the cases were performed due to obstetric indication only not directly related to COVID status of the patient (Figure 2).

Out of 8 patients who succumbed due to covid infection in pregnancy, 6 belonged to 25-34 week gestation which is $75 \%$. Whereas, all the patients infected in $1^{\text {st }}$ trimester recovered, out of 38 symptomatic patients in $>34$ weeks gestational age group 37 patients recovered (97.3\%), only 1 succumbed to COVID.

9 patents were having comorbid conditions needed hospitalisation in ICU.

Table 1: Relation of age with symptoms.

\begin{tabular}{|llllll|}
\hline \multirow{2}{*}{ Age (years) } & Symptom (30) & \multicolumn{2}{l|}{ Asymptomatic (45) } & Total \\
\hline $\mathbf{2 0}$ & Recovered & Death & Recovered & Death & 2 \\
\hline $\mathbf{2 1 - 3 5}$ & 0 & 1 & 1 & 0 & 61 \\
\hline$>\mathbf{3 5}$ & 20 & 2 & 39 & 0 & 12 \\
\hline Total & 2 & 5 & 5 & 0 & 75 \\
\hline
\end{tabular}

Table 2: Gestational age in relation to symptoms.

\begin{tabular}{|llll|}
\hline GA (weeks) & Asymptomatic & Symptomatic & Total \\
\hline$<\mathbf{1 2}$ & 5 & 4 & 9 \\
\hline $\mathbf{1 2 - 2 4}$ & 5 & 3 & 8 \\
\hline $\mathbf{2 5 - 3 4}$ & 10 & 9 & 19 \\
\hline$>\mathbf{3 4}$ & 25 & 14 & 39 \\
\hline Total & 45 & 30 & 75 \\
\hline
\end{tabular}

Table 3: Management.

\begin{tabular}{|lllll|}
\hline Conservative & S\&E & VD/expulsion & LS-CS & Others (laparotomy) \\
\hline $\mathbf{8}$ & 9 & 18 & 39 & 1 \\
\hline
\end{tabular}




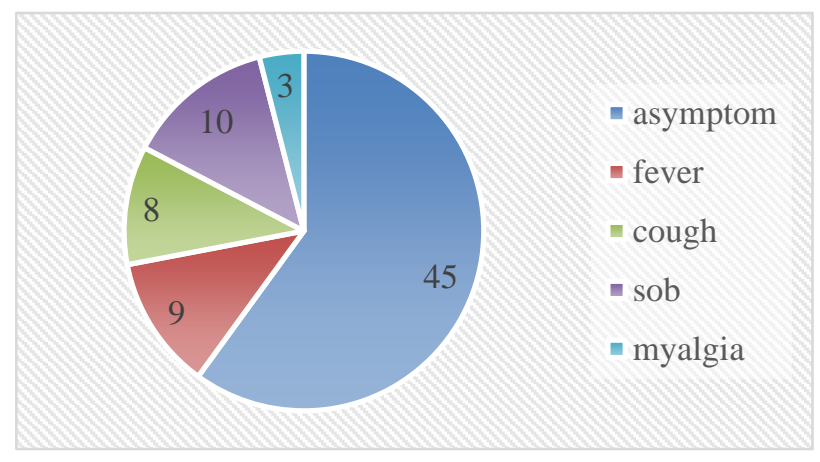

Figure 1: Symptoms.

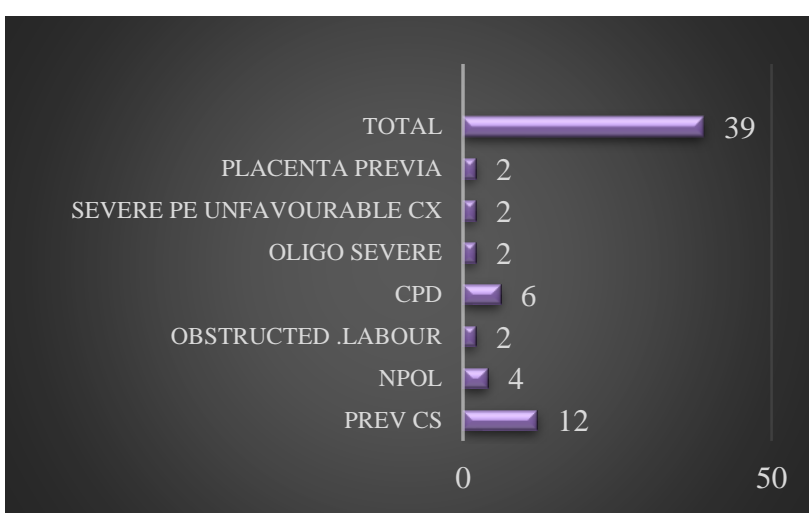

Figure 2: Indication for LSCS.

Table 4: Maternal outcome in relation to gestational age.

\begin{tabular}{|lll|}
\hline GA (weeks) & Recovered & Death \\
\hline$<\mathbf{1 2}$ & 9 & 0 \\
\hline $\mathbf{1 2 - 2 4}$ & 6 & 1 \\
\hline $\mathbf{2 5 - 3 4}$ & 13 & 6 \\
\hline$>\mathbf{3 4}$ & 37 & 1 \\
\hline
\end{tabular}

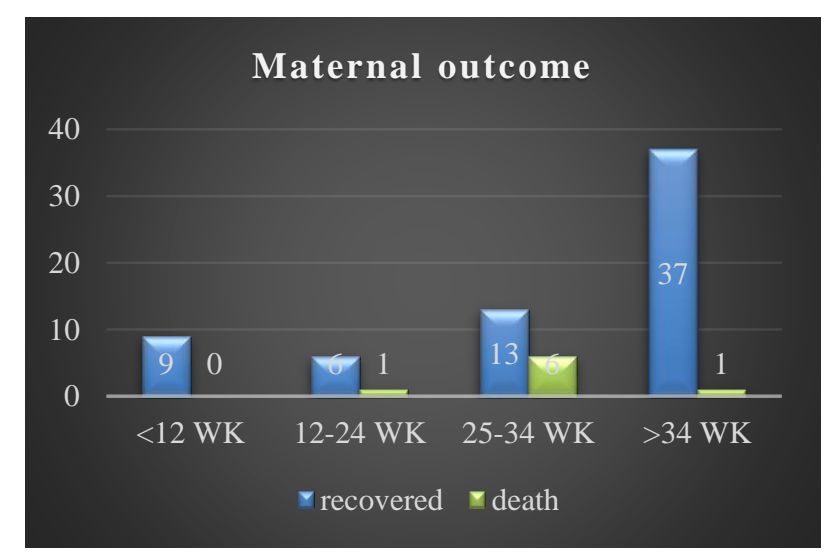

Figure 3: Maternal outcome.

Out of 75 patients 9 patients were having various comorbidities during pregnancy which includes diabetes (2), hypertension (3), thalassemia (1), bronchial asthma (1), chronic hepatitis (1), and hypothyroidism (1). 8 out of
As per Table 5, on admission, total leucocyte count (TLC) was increased in $84 \%(63 / 75)$ of the patients and neutrophil/lymphocyte ratio (NLR) was elevated in $72 \%$ (54/75) of patients. Elevated levels of C-reactive protein were found in $54 \%$ of the patients. In view of increased TLC, injection methyl-prednisone was administered alongside monitoring blood sugar level and found to be very effective in controlling the inflammation. Patients with severe disease had more prominent laboratory abnormalities than those with mild disease. In the symptomatic cases that underwent high-resolution computed tomography (HRCT) thorax (antenatal patients with lead shield) at the time of admission, 20\% (15/75) revealed abnormal results ( 1 bilateral pleural effusion and 14 showed patchy haziness and peripheral opacities) and $34.7 \%$ (26/75) reported increased serum levels of LDH.

Table 5: Investigations.

\begin{tabular}{|l|l|}
\hline Investigations & No. of patients \\
\hline TLC & 63 \\
\hline D dimer & 28 \\
\hline CRP & 41 \\
\hline LDH & 26 \\
\hline CT & 15 \\
\hline
\end{tabular}

There were total 57 deliveries in this study among which $11(19.3 \%)$ were IUFD (46 live born and 11 IUFD deliveries). All the $18(31.5 \%)$ live born preterm neonates were admitted to the NICU for further treatment due to respiratory distress syndrome and to rule out COVID-19 infection. The incidence of preterm birth before 37 weeks was $26.3 \%$ (15/57). $96.9 \%$ of neonates were tested for SARS-CoV-2 viral nucleic acid on nasopharyngeal and pharyngeal samples and $17.5 \%(10 / 57)$ were resulted positive.

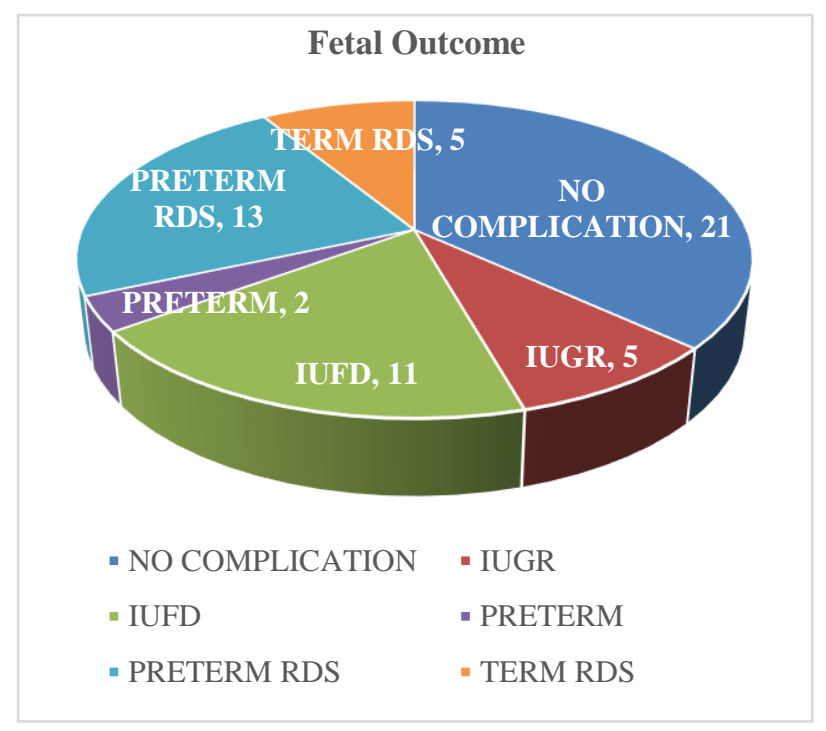

Figure 4: Fetal outcome.

All these cases underwent necessary laboratory and radiological investigations and detailed epidemiologic 
history. All the full term pregnant women were motivated, counselled, explained the daily fetal movement counting (DFMC), undergone ultrasonography (USG) fetus including fetal Doppler study on admission. Antibiotics and supportive multivitamins including vitamin $\mathrm{C}$ were given in all the 75 patients as per ICMR guidelines.

\section{DISCUSSION}

In this preliminary study of 75 cases, we observed that the clinical characteristics of the women with COVID-19 infection during pregnancy were similar to those of nonpregnant adults with COVID-19 infection, comparable with studies by Guan et al and $\mathrm{Wu}$ et al. ${ }^{15,16}$ Data from 75 cases, with a total of 30 symptomatic women with ongoing pregnancy, indicated that the most common symptoms at presentation were cough (60\%) and myalgia (50\%); 45 of pregnant patients did not present with symptoms, but most of these patients were diagnosed as having COVID-19 infection by intensive testing and contact tracing during the initial phase of outbreak in India. 54 out of 75 cases, two-third of the patients had lymphopenia (increased NLR) and increased C-reactive protein, and 20\% (15 cases) showed patchy haziness and peripheral opacities in HRCT thorax. We observed clinical, laboratory, and radiologic characteristics that are similar to published pregnant and non-pregnant cases of COVID-19 infection. ${ }^{15,16}$ In normal pregnancy there is physiological activation of the innate limb of the immune response but study by Nacaasha et al reported that pregnant women with acute infection display a more aggressive immune respone. ${ }^{18}$ In present study, 9 of 75 (12\%) pregnant women experienced severe COVID-19 infection, which is comparable to the $15.6 \%$ of severe disease that has been reported in studies from China. ${ }^{11,12}$ Immunomodulators like IL-6 inhibitors-tocilizumab has been used for treating selected cases of severe pneumonia in ICU admitted patients in study institute but has not been administered in any of the 75 cases in this study. Study by Alattar et al reported that use of tocilizumab in patients with severe COVID-19 was associated with dramatic decline in inflammatory markers, radiological improvement and reduced ventilatory support requirements. ${ }^{19}$ It has been reported that viral pneumonia in pregnant women is associated with an increased risk of preterm birth, fetal growth restriction (FGR), and perinatal mortality by Madinger et al. ${ }^{20}$ It is reported by Chen et al that pregnant patients infected with viral pneumonia other than SARSCoV-2 have increased risk of fetal growth restriction (FGR), preterm delivery, low birthweight and Apgar score $\leq 7$ at 5 minutes. ${ }^{21}$ These findings correspond with our study. A case series of 12 COVID-19 infected pregnant women in Hong Kong reported 3 maternal deaths; 4 of 7 patients $(57 \%)$ had spontaneous abortion in first trimester, 4 of 5 patients $(80 \%)$ had preterm birth, and 2 pregnant women recovered but their ongoing pregnancies were complicated by FGR. ${ }^{22}$

It is reported from this study that the risk of spontaneous abortion is not increased in pregnant women infected with
SARS-CoV-2 from the background risk of the healthy pregnant women. ${ }^{23}$ This study also suggests that, although the risk of any preterm birth before 37 weeks gestation is increased, COVID-19 is not associated with an increased risk of spontaneous preterm birth before 37 weeks. There were 15 cases of preterm birth (12 by cesarean delivery, and 2 preterm vaginal delivery) due to obstetric indication. $\mathrm{RCOG}$, in consultation with the $\mathrm{RCPCH}$, have provided guidance for delivery and neonatal care, which recommends that mode of delivery be determined primarily by obstetric indication, and recommends against routine separation of COVID-19-affected mothers and their babies. ${ }^{11}$ One of the main purposes of this study was to look for the possibility of vertical transmission of SARS-CoV-2 infection. We could only evaluate neonatal nasopharyngeal and pharyngeal swab samples at birth to ascertain the possibility of vertical transmission. This study results indicated that SARS-CoV-2 was positive in $10(17.5 \%)$ neonates at birth suggesting that there could be possibility of intrauterine fetal infection because of SARSCoV-2 infection during the $2^{\text {nd }}$ or $3^{\text {rd }}$ trimester of pregnancy. This study findings are in agreement with 2 recent research letters reported on 3 neonates born to women with confirmed COVID-19 who tested positive for immunoglobulin $\mathrm{G}$ and immunoglobulin $\mathrm{M}$ antibodies despite having a negative viral nucleic acid result, raising the possibility of vertical transmission, but more data are needed. ${ }^{24,25}$ There is controversy between guidance for delayed cord clamping, which is due to lack of evidence. Consensus guidance from China advises that 'delayed cord clamping is not recommended', in order to reduce the risk of vertical transmission, and that infants should be separated from mothers affected by COVID-19. ${ }^{26}$ Delayed cord clamping is not practiced in our institute and this was same for deliveries of COVID-19 infected pregnant women. Interim guidance from ISUOG advises clinicians to consider not undertaking delayed cord clamping. ${ }^{27}$ However 1 min delayed cord clamping is very unlikely to affect the risk of vertical transmission due to fact that there is already enough exposure to maternal secretions and blood during delivery. Infants may contract infection after delivery from their mothers through normal mode of transmission. All the neonates in this study were top-fed in NICU with expressed breastmilk. WHO guidelines states that 'the benefits of skin-to-skin contact and breastfeeding substantially outweigh the potential risks of transmission and illness associated with COVID-19 infection.while guidance from China states that 'Infants shouldn't be given feeding with the expressed breast milk from confirmed or suspected COVID-19 mothers'. ${ }^{28}$

\section{Limitations}

Effects of COVID-19 on pregnant women during first and second trimester were not clear. This study only assessed pregnant women during hospitalisation, but long term effect on liver and other organs as well as newborn require further studies. We couldn't retest the new-born again after 24 hours. 


\section{CONCLUSION}

COVID-19 in pregnancy with or without any comorbidities can cause complications to both mother and fetus irrespective of treatment particularly if the infection occurs in pregnancy. The impact of COVID-19 on maternal and new born health is unclear. We aimed to evaluate the association between severe SARS-CoV-2 infection during pregnancy and adverse pregnancy outcomes. In pregnancy, due to the physiological alterations in cardiovascular, respiratory and immune system, the pregnant women are at greater risk and vulnerable to become the victims of pathogens, especially in the case of outbreak like SARS-CoV-2. Currently, there is no evidence that pregnant women are at greater risk to succumb to COVID-19 infection and experience severe pneumonia. This preliminary study however does not rule out the possibility of vertical transmission of SARS-CoV2 when it manifests towards the end of pregnancy. This study does not report any increased risks of spontaneous abortion and spontaneous preterm deliveries. Currently there is no definite data regarding the risk of teratogenicity, congenital infection, labour management and mode of delivery. Early data analysis regarding the pregnancy outcomes in COVID-19 are optimistic but due to the limited availability of study in the literature on this disease in pregnancy and restricted sample size, there is a need for systematic data collection and follow-up of disease in pregnancy. This would guide us better regarding the management of pregnant women with covid infection. The life of health care professionals engaged in the battle against SARS-CoV-2 is equally important and adequate safety measures were ensured by using fully equipped personal protective equipment (PPE) kits including N-95 masks.

\section{Funding: No funding sources}

Conflict of interest: None declared

Ethical approval: The study was approved by the Institutional Ethics Committee

\section{REFERENCES}

1. Zhu N, Zhang D, Wang W, Li X, Yang B, Song J, et al. China novel coronavirus investigating and research team. A novel coronavirus from patients with pneumonia in China, 2019. N Engl J Med. 2020;382:727-33.

2. Weiss SR, Leibowitz JL. Coronavirus pathogenesis. Adv Virus Res. 2011;81:85-6.

3. Masters PS, Perlman S. Coronaviridae. In: Knipe DM, Howley PM, editors. Fields virology. 6th edition. Lippincott Williams and Wilkins. 2013:825-58.

4. Tang P, Wang J, Song Y. Characteristics and pregnancy outcomes of patients with severe pneumonia complicating pregnancy: a retrospective study of 12 cases and a literature review. BMC Pregnancy and Childbirth. 2018;18(1).
5. Wenling Y, Junchao Q, Xiao Z, Ouyang S. Pregnancy and COVID-19: management and challenges. Rev Inst Med Trop Sao Paulo. 2020;62.

6. Dorélien A. The effects of in utero exposure to influenza on birth and infant outcomes in the US. Popul Dev Rev 45: 489-523, 2019. doi:10.1111/padr.12232. [PMC free article] [PubMed] [CrossRef] [Google Scholar]

7. Mosby LG, Rasmussen SA, Jamieson DJ. 2009 pandemic influenza A (H1N1) in pregnancy: a systematic review of the literature. Am J Obstet Gynecol. 2011;205:10-8.

8. Su S, Wong G, Shi W, Liu J, Lai AC, Zhou J, et al. Epidemiology, genetic recombination, and pathogenesis of coronaviruses. Trends Microbiol. 2016;24:490-502.

9. Cui J, Li F, Shi ZL. Origin and evolution of pathogenic coronaviruses. Nat Rev Microbiol. 2019; 17:181-92.

10. Chan JF, Kok KH, Zhu Z, Chu H, To KK, Yuan S, et al. Genomic characterization of the 2019 novel human-pathogenic coronavirus isolated from a patient with atypical pneumonia after visiting Wuhan. Emerg Microbes Infect. 2020;9:221-36.

11. Royal College of Obstetricians Gynaecologists, Royal College of Midwives. Coronavirus infection in pregnancy: Information for healthcare professionals. 2020. Available at: https://www.rcog.org.uk/global. Accessed on 13 April 2021.

12. Centers for Disease Control and Prevention. Coronavirus Disease (COVID-19). People who need extra precautions: Pregnancy and Breastfeeding. Available at: https://www.cdc.gov/coronaviru. Accessed on 13th April 2020.

13. Hui DS, Zumla A. Severe acute respiratory syndrome: historical, epidemiologic, and clinical features. Infect Dis Clin. 2019;33(4):869-89.

14. Silasi M, Cardenas I, Kwon JY, Racicot K, Aldo P, Mor G. Viral infections during pregnancy. Am J Reprod Immunol. 2015;73(3):199-213.

15. Schwartz DA, Graham AL. Potential maternal and infant outcomes from (Wuhan) coronavirus 2019nCoV infecting pregnant women: lessons from SARS, MERS, and other human coronavirus infections. Viruses. 2020;12(2):194.

16. Maxwell C, McGeer A, Tai KFY, Sermer M. No. 225Management guidelines for obstetric patients and neonates born to mothers with suspected or probable Severe Acute Respiratory Syndrome (SARS). J Obstet Gynaecol Canada. 2017;39(39):130-7.

17. Allotey J, Stallings E, Bonet M, Yap M, Chatterjee S, Kew T, et al. Clinical manifestations, risk factors, and maternal and perinatal outcomes of coronavirus disease 2019 in pregnancy: living systematic review and meta-analysis. BMJ (Clinical research ed). 2020;370:3320.

18. World Health Organization. Laboratory testing for 2019 novel coronavirus (2019-nCoV) in suspected human cases. Interim guidance. Available at: https://www.who.int/publications-detail/laboratory- 
testing-for-2019-novel-coro-navirus-in-suspectedhuman-cases-20200117. Accessed on 24 March 2020.

19. Ministry of health and family welfare. Revised discharged policy, dated 8th May, 2020. Available at: https://www.mohfw.gov.in/pdf/FAQsonRevisedDisc hargePolicy.pdf. Accessed on 10 June 2020.

20. Guan WJ, Ni ZY, Hu Y, Liang WH, Ou CQ, He JX, et al. Clinical characteristics of coronavirus disease 2019 in China. N Engl J Med. 2020;382(18):1708-20.

21. Wu Z, McGoogan JM. Characteristics of and important lessons from the coronavirus disease 2019 (COVID-19) outbreak in China: summary of a report of 72314 cases from the Chinese center for disease control and prevention. JAMA. 2020;323(13):123942.

22. Chawla D, Chirla D, Dalwai S, Deorari AK, Ganatra A, Gandhi A, et al. Perinatal-neonatal management of COVID-19 infection-guidelines of the federation of obstetric and gynaecological societies of India (FOGSI), National neonatology forum of India (NNF), and Indian Academy of Pediatrics (IAP). Indian Pediatr. 2020;57(6):536-48.

23. Madinger NE, Greenspoon JS, Ellrodt AG. Pneumonia during pregnancy: has modern technology improved maternal and fetal outcome? Am J Obstet Gynecol. 1989;161:657-62.

24. Chen YH, Keller J, Wang IT, Lin CC, Lin HC. Pneumonia and pregnancy outcomes: a nationwide population-based study. Am J Obstet Gynecol. 2012;207:288

25. Dashraath P, Wong JLJ, Lim MXK. Coronavirus disease 2019 (COVID-19) pandemic and pregnancy. Am J Obstet Gynecol. 2020;222:521-31.

26. Knight M, Bunch K, Vousden N, Morris E, Simpson $\mathrm{N}$, Gale C, et al. Characteristics and outcomes of pregnant women admitted to hospital with confirmed SARS-CoV-2 infection in UK: national population based cohort study. BMJ (Clinical research ed). 2020;369:2107.
27. Wong SF, Chow KM, Leung TN, Ng WF, Ng TK, Shek CC, et al. Pregnancy and perinatal outcomes of women with severe acute respiratory syndrome. Am J Obstet Gynecol. 2004;191:292-7.

28. Al Wattar B, Murugesu N, Tobias A, Zamora J, Khan KS. Management of first-trimester miscarriage: a systematic review and network meta-analysis. Hum Reprod Update. 2019;25:362-74.

29. Zeng H, Xu C, Fan J. Antibodies in infants born to mothers with COVID-19 pneumonia. JAMA. 2020;323(18):1848-9.

30. Dong L, Tian J, He S, Zhu C, Wang J, Liu C, et al. Possible vertical transmission of SARS-CoV-2 from an infected mother to her newborn. JAMA. 2020;323(18):1846-8.

31. Wang L, Shi Y, Xiao T, Fu J, Feng X, Mu D, et al. Working committee on perinatal and neonatal management for the prevention and control of the 2019 novel coronavirus infection. Chinese expert consensus on the perinatal and neonatal management for the prevention and control of the 2019 novel coronavirus infection (First edition). Ann Transl Med. 2020;8:1-8.

32. Poon LC, Yang H, Lee JCS, Copel JA, Leung TY, Zhang Y, et al. ISUOG Interim Guidance on 2019 novel coronavirus infection during pregnancy and puerperium: information for healthcare professionals. Ultrasound Obstet Gynecol. 2020;55:700-8.

33. World Health Organization. Frequently asked questions: Breastfeeding and COVID-19. 2020. Available at: https://www.who.int/docs/defaultsource/maternal-health/faqs-breastfeeding-andcovid-19.pdf?sfvrsn=d839e6c0_5. Accessed on 10 June 2021.

Cite this article as: Dash P, Padhi SK, Behera RN. Observational study of fetomaternal outcome in pregnancy with COVID-19 infection. Int J Reprod Contracept Obstet Gynecol 2022;11:752-8. 